This is a self-archived version of an original article. This version may differ from the original in pagination and typographic details.

Author(s): Yin, F.; Akola, Jaakko; Koskinen, Pekka; Manninen, Matti; Palmer, R.E.

Title: Bright beaches of nanoscale potassium islands on graphite in STM imaging,

Year: 2009

Version: Published version

Copyright: ㄷ 2009 The American Physical Society

Rights: In Copyright

Rights url: http://rightsstatements.org/page/InC/1.0/?language=en

Please cite the original version:

Yin, F., Akola, J., Koskinen, P., Manninen, M., \& Palmer, R.E. (2009). Bright beaches of nanoscale potassium islands on graphite in STM imaging,. Phys. Rev. Lett., (102), 106102.

https://doi.org/10.1103/PhysRevLett.102.106102 


\title{
Bright Beaches of Nanoscale Potassium Islands on Graphite in STM Imaging
}

\author{
F. Yin, ${ }^{1}$ J. Akola, ${ }^{2,3}$ P. Koskinen, ${ }^{2}$ M. Manninen, ${ }^{2}$ and R. E. Palmer ${ }^{1}$ \\ ${ }^{1}$ Nanoscale Physics Research Laboratory, School of Physics and Astronomy, University of Birmingham, Edgbaston, \\ Birmingham, B15 2TT, United Kingdom \\ ${ }^{2}$ Nanoscience Center, Department of Physics, University of Jyväskylä, FI-40014 Jyväskylä, Finland \\ ${ }^{3}$ Department of Physics, Tampere University of Technology, FI-33101 Tampere, Finland
}

(Received 22 December 2008; published 9 March 2009)

\begin{abstract}
We demonstrate, via scanning tunneling microscopy (STM) measurements performed at $48 \mathrm{~K}$, the existence of "bright beaches" at the edges of K islands (diameter $\sim 5-500 \mathrm{~nm}$ ) on the graphite surface. The enhanced tunneling current is only observed in monolayer-high islands on graphite, and not in islands of similar geometry on top of a K monolayer film. First-principles density functional calculations and STM simulations suggest that this is an STM field effect, which appears as the positive tip attracts donated electrons back to the metallic $\mathrm{K}$ islands. The restored charge accumulates preferentially at the island edges.
\end{abstract}

DOI: 10.1103/PhysRevLett.102.106102

The interaction of alkali metals with the graphite surface remains a topic of active research nearly two decades after the discovery of the Wigner-like repulsive dispersed phase [1], for three reasons. First, they are model systems for the study of chemisorption [2]. Second, the alkali-graphite intercalation compounds have technological relevance to, e.g., catalysts, superconductors, solid-state Li ion batteries, and solid-state hydrogen reservoirs [3-8]. Third, alkali adsorption on graphene monolayers is a way to expose the Fermi-level band structure in photoemission measurements [9], so focusing attention on the corresponding parent systems.

The adsorption of potassium on graphite has now been studied extensively by both structural and spectroscopic techniques [10-12]; theoretical approaches include band structure and cluster calculations [2,10,13-16]. The results confirm the development of the K-graphite phase diagram (at temperatures of $\sim 90 \mathrm{~K}$ ) from a dispersed, repulsive phase of isolated $\mathrm{K}$ atoms at very low coverage (driven by $\mathrm{K}$ charge transfer to the surface) through the nucleation (and coexistence) of close-packed $(2 \times 2) \mathrm{K}$ islands to a complete $\mathrm{K}$ monolayer. However, local measurements of the system are notable by their absence. Specifically, STM images of $\mathrm{K}$ on graphite have not been reported, unlike alkali-graphite intercalation compounds [17], alkali adsorption on semiconductors [18], and sodium films on graphite [19]. Here, we employ the STM to demonstrate, in real space, the existence of "bright beach" features around the rim of $\mathrm{K}$ islands with diameters of 5-500 nm; theoretical calculations and STM simulations indicate the phenomenon is caused by STM tip field-induced, spatially dependent restoration of charge (initially donated by the islands to the surface) in these finite systems.

The experiments were performed in an ultra high vacuum (UHV) chamber with a base pressure of $6 \times$ $10^{-11}$ mbar. Potassium was evaporated from a thermal getter source (SAES) at a surface temperature of $90 \mathrm{~K}$.
PACS numbers: 68.37.Ef, 61.46.-w, 71.20.Dg, 73.20.-r

The (highly oriented pyrolytic) graphite was cleaved with tape just before loading into the UHV chamber, where it was annealed to $450{ }^{\circ} \mathrm{C}$. Such surfaces display clean, atomically flat terraces in STM imaging. We did not observe stable STM images of submonolayer coverages of $\mathrm{K}$-graphite at $90 \mathrm{~K}$, so imaging was conducted after cooling to $48 \mathrm{~K}$. The STM employed was an Omicron VT-STM with a Pt/Ir (90/10) tip.

Figure 1(a) presents the STM topography of the graphite surface (surface bias voltage $-2 \mathrm{~V}$, tunneling current $40 \mathrm{pA}$ ) at $48 \mathrm{~K}$ with a $\mathrm{K}$ adatom coverage, $\theta$, of 0.86 monolayers (ML). The $\mathrm{K}$ coverage is defined as $\theta=$ $\Sigma S_{i} / S_{a}$, where $S_{i}$ represents the surface area covered by a K island and $S_{a}$ is the whole area of surface scanned by the STM; thus, $\theta=1 \mathrm{ML}$ refers to a close-packed K monolayer completely covering the graphite surface. The orientation of these $\mathrm{K}$ clusters with respect to the graphite surface will be treated elsewhere [20]. Based on the original electron energy loss spectroscopy (EELS) and low energy electron diffraction (LEED) work [1], we should see a mixture of low density correlated $(7 \times 7)$ gas phase and (primarily) islands of the close-packed $\mathrm{K}(2 \times 2)$ phase at this coverage [21]. In practice, the gas phase is very difficult to image with the STM tip, while a few secondlayer $\mathrm{K}$ islands are observed in addition to the heavily faceted, monolayer islands of $\mathrm{K}$ in Fig. 1(a). The experimental height of these islands is $5.6 \AA$, rather close to the lattice constant of bulk K (5.33 $\AA$ ) [22], confirming they are single layers. The top of the islands in Fig. 1(a) is more or less flat, although there is a hint of the bright ring around the edges of the islands which becomes much more apparent when the surface bias voltage is changed to $-4 \mathrm{~V}$, Fig. 1(b). The bright beach features observed in Fig. 1(b) are not seen when the sample bias voltage is positive.

Figure 1(c) is set out to show how the brightness of the beach depends upon tip-surface bias voltage in the STM junction. Plotted is the apparent height of the rim of the 

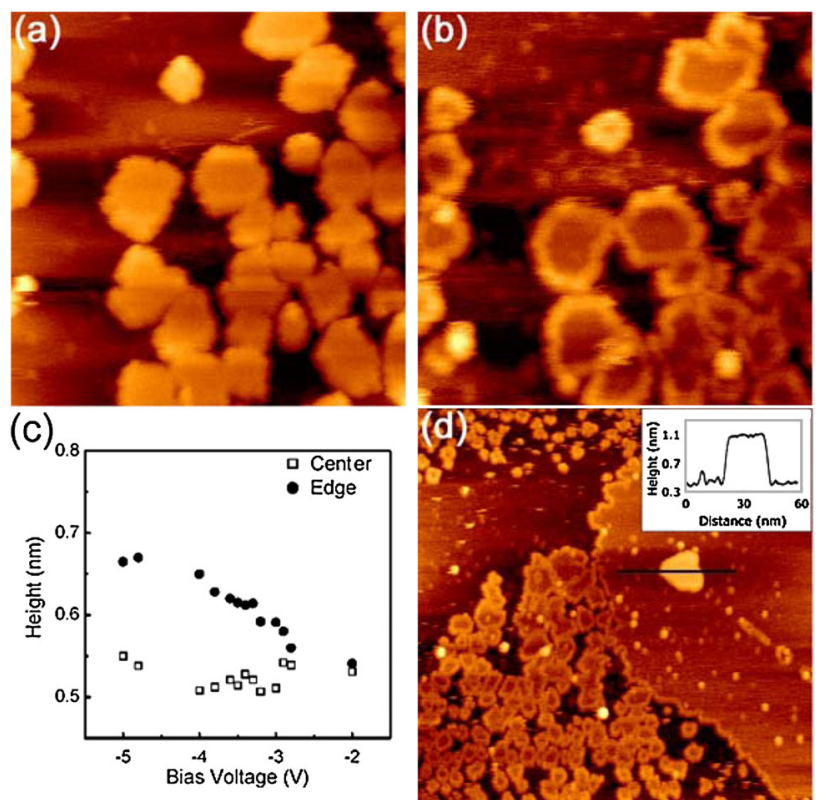

FIG. 1 (color online). (a) Constant current STM image $(57 \mathrm{~nm} \times 57 \mathrm{~nm})$ of $\mathrm{K}$ islands on graphite at a surface coverage of $\theta=0.86$ monolayers (surface bias voltage, $-2.0 \mathrm{~V}$; tunnel current $40 \mathrm{pA}$ ). (b) As (a) except the surface bias voltage is $-4 \mathrm{~V}$. (c) The apparent (average) heights of the edges and centers of the $\mathrm{K}$ islands (relative to the graphite surface) as a function of (negative) surface bias voltage. (d) STM image $(200 \mathrm{~nm} \times 200 \mathrm{~nm})$ showing a region of the surface which contains both $\mathrm{K}$ monolayer islands on graphite and a $\mathrm{K}$ monolayer island on top of an extended $\mathrm{K}$ monolayer (surface bias voltage, $-4.6 \mathrm{~V}$; tunnel current, $40 \mathrm{pA}$ ); inset, line profile of the second-layer island.

islands as a function of (negative) surface bias voltage, together with the height of the island center. For bias voltages more negative than $-2.8 \mathrm{~V}$, the apparent height difference between the "beach" and the interior of the island is evident; the effect saturates at $-4 \mathrm{~V}$, with a value well over $1 \AA$. Of course, this is not to propose that the atomic edge of the $\mathrm{K}$ island is "really" higher than the center; electronic effects may dominate in the STM, as the dependence on bias voltage shows.

We also found that, although the bright beach features are only dimly visible with a standard tip for surface bias voltages less negative than $-2.8 \mathrm{~V}$, Fig. 1(a), the pickup by the tip of (presumably $\mathrm{K}$ ) atoms from the surface makes the bright beaches readily visible at all (negative) voltages. The difference between the images obtained before, Fig. 2(a), and after, Fig. 2(b), K pickup is evident. Moreover, the height difference between the beach and island center is in these circumstances no longer so strongly dependent on surface bias voltage. The collection of surface atoms by the STM tip is a standard "trick" to obtain STM images with improved lateral spatial resolution [23].

What is the origin of the bright beach features? Is it a geometrical or an electronic effect? If the latter, does it
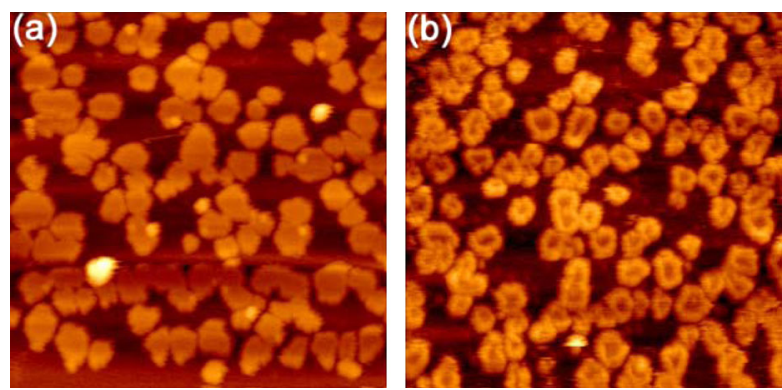

FIG. 2 (color online). A pair of constant current STM images $(102 \mathrm{~nm} \times 102 \mathrm{~nm})$ of $\mathrm{K}$ islands on graphite (a) before and (b) after the tip picks up atoms (presumed $\mathrm{K}$ atoms) from the surface (surface bias voltage $-2.0 \mathrm{~V}$; tunnel current, $40 \mathrm{pA}$ ).

arise purely from lateral confinement of electrons in the finite $\mathrm{K}$ island, say, or does it depend on the interaction between the $\mathrm{K}$ island and the graphite support? Is it related to the STM imaging process itself? Consider first one final piece of (highly illuminating) experimental evidence. In Fig. 1(d), we show a region of the surface in which an "archipelago" of $\mathrm{K}$ islands runs up to a piece of dry land, i.e., the dense monolayer of $\mathrm{K}$ on the right side of the image. Notable features of the land mass are (a) the bright beaches along the coastline (which implies they are associated with edges, not lateral confinement across islands) and (b) the small bright features on top of the land mass, and especially the faceted $\mathrm{K}$ island (line scan inset) which sits one monolayer high on top of the extended $\mathrm{K}$ monolayer. An outer rim of increased apparent height (i.e., a bright beach) is never observed in $\mathrm{K}$ islands that sit on top of a $\mathrm{K}$ layer, striking evidence that the phenomenon arises from the interaction (bonding) between the $\mathrm{K}$ islands and the graphite surface.

In order to probe the origin of the beach features more deeply, we modeled two monolayer $\mathrm{K}$ islands on graphite with density functional theory (DFT). First, the CPMD program [24] was used to optimize the geometry of $\mathrm{K}$ islands on top of two layers of graphene ( $A B$ stacking). We used the recent PBEsol approximation for the exchange-correlation energy [25], Troullier-Martins pseudopotentials ( $\mathrm{K}$ valence $3 p^{6} 4 s$ ) [26], and a plane wave basis set with a 70 Ry kinetic energy cutoff. Second, electronic structure analysis and STM simulations were performed with the GPAW code [27], which uses realspace grids (0.2 $\AA$ grid spacing) and the projectoraugmented wave formalism [28]. Because of the large unit cell sizes incorporating hundreds of carbon atoms, a single $\boldsymbol{k}$ point in the Brillouin zone was sufficient at both stages of calculation.

Our model islands are a hexagonal $\mathrm{K}_{19}$ cluster of diameter $2.0 \mathrm{~nm}$ and a $\mathrm{K}_{24}$ strip of width $2.7 \mathrm{~nm}$, Figs. 3(a) and 3(b). Both islands have a vertical height of 2.95-3.15 above the graphite surface, and they conform to a $(2 \times 2)$ atomic arrangement on the surface. The structure optimization shrinks the $\mathrm{K}-\mathrm{K}$ bond lengths involving edge atoms 

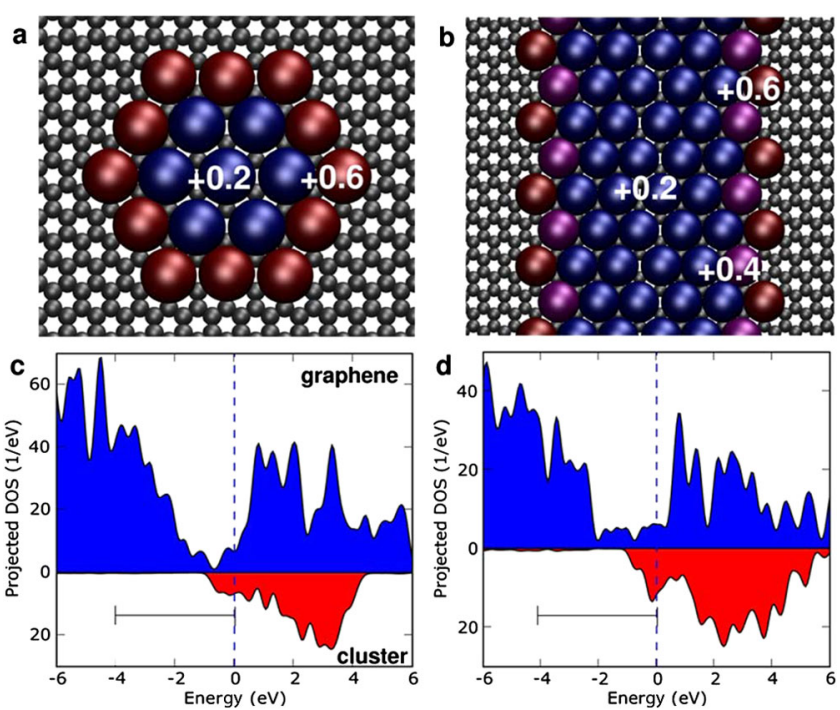

FIG. 3 (color online).

(a) $\mathrm{K}_{19}$ cluster and (b) $\mathrm{K}_{24}$ strip (more than one unit cell shown) on graphite. Numbers stand for the Bader charges of $\mathrm{K}$ atoms. Total charge for the cluster is $+7.9 e$ and for the strip $+8.5 e$. Projected density of Kohn-Sham states for (c) cluster and (d) strip. The Fermi-level is set to zero, and the horizontal bar denotes the bias window used in the STM simulations of Fig. 4.

by $0.2 \AA$, and drags the edge atoms down towards the graphite surface. The interior parts of the islands are charged by $+0.2 e$ per $\mathrm{K}$ atom, whereas the edges are charged as much as $+0.6 \mathrm{e} /$ atom, according to the Bader charge analysis. The average charge transfer is $+0.3 \mathrm{e} / \mathrm{K}$; a value $+0.11 \mathrm{e} / \mathrm{K}$ was reported for a full $\mathrm{K}$ monolayer [2]. The electronic structure of the $\mathrm{K}$ islands displays behavior characteristic of a 2D free-electron metal (jellium model [29]), and the adsorbate-substrate interaction can be characterized as primarily ionic [2].

The projections of the electronic density of states (DOS) shown in Figs. 3(c) and 3(d) support the concept of charge transfer from the $\mathrm{K}$ islands to the graphite surface. The graphene conduction band states become occupied as the zero band gap is shifted below the Fermi-level. The band of states corresponding to the delocalized $\mathrm{K} 4 s$ valence electrons starts at around $-1.0 \mathrm{eV}$, which implies that an STM bias more negative than $-1.0 \mathrm{~V}$ already collects electrons tunneling from all the relevant states of the $\mathrm{K}$ islands.

STM simulations of the $\mathrm{K}$ islands produced using the Tersoff-Hamann theory [30] are shown in Figs. 4(a) and 4(c). Here, the STM current is associated with the local density of states; this is the electron density of Kohn-Sham states within the bias window, referenced to the Fermi energy. Such STM simulations do not agree with the experiment: neither the STM topographies in Figs. 4(a) and 4(c) nor the STM line scans in Figs. 4(e) and 4(g) show any pronounced brightness at the island edges. The STM height of the $\mathrm{K}$ islands $(\sim 5.1 \AA$, measured from the graphene plane) is also lower than the experimental one (5.6 A). This disagreement is hardly surprising since the islands are positively charged and the edges lose even more electrons than the central regions; i.e., the discrepancy between experiment and simulation arises directly from the self-consistent electronic structure.

To resolve the discrepancy between experiment and theory and thus the origin of the bright beach features, we recall that the charge transfer from the alkali islands to the surface builds up an electric field between the $\mathrm{K}$ islands and graphene layers until the resulting electrostatic energy term balances the chemical energy of bonding. Let us now reverse this argument: application of an external electric field should be able to control the charge transfer. Thus, a positive tip (negative sample bias) will provide an electric field pointing (by convention) towards the surface which can attract electrons back to a $\mathrm{K}$ island located beneath the tip from the underlying graphite. To simulate the effect of such a field, we solved the electronic structure in the presence of a homogeneous electric field of magnitude $0.5 \mathrm{~V} / \AA$ perpendicular to the surface (corresponding to a
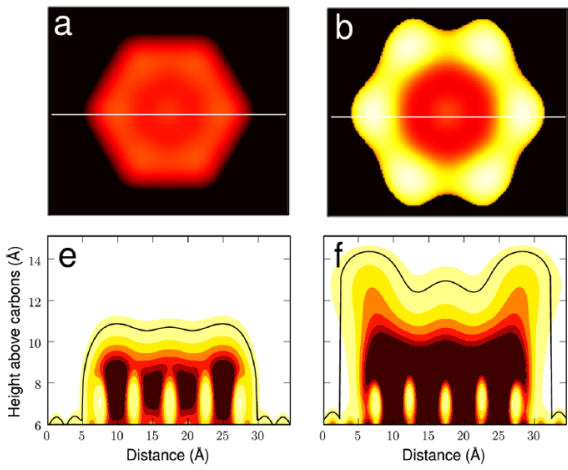
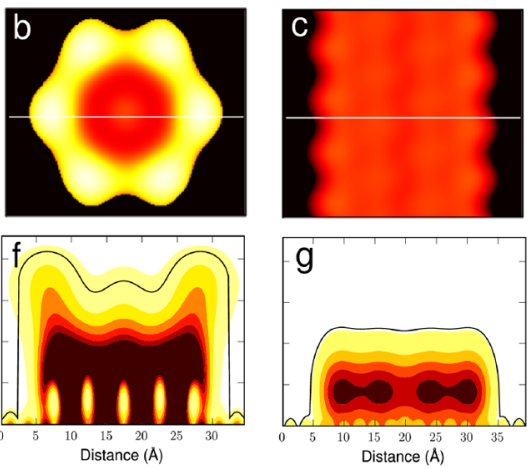

FIG. 4 (color online). Simulated STM images for $\mathrm{K}_{19}$ cluster (a) and (b) and $\mathrm{K}_{24}$ strip (c) and (d) without [(a), (c)] and with [(b), (d)] external electric field $(0.5 \mathrm{~V} / \AA$, pointing downwards). Line scans and cross sections [locations shown by lines in (a)-(d)] of the local electron density for cluster (e) and (f) and strip (g) and (h) without and with the field. Zero height of the line scans (solid black curves) is at the level of carbon atoms, and the $\mathrm{K}$ atoms appear at $2.95-3.15 \AA$. Bias voltage is $-4.0 \mathrm{~V}$, and the STM images and line scans are produced with electron density $n=4 \times 10^{-4} \AA^{-3}$. 
bias voltage of 2-3 V). A homogeneous field is justified because a typical radius of curvature $(R)$ for an STM tip is much larger than the tip height $(h)$ above the surface $(R \sim 1000 \AA, h \sim 5 \AA)$ [30]. A more realistic tip field model is difficult as the field depends on the tip geometry and does not simply "penetrate" into the system. Nevertheless, our approximation should capture the essential features.

The outcome of these simulations is a field-induced restoration of electron density at the island edges, as seen in Figs. 4(b) and 4(d). The field attracts $\sim 4$ electrons to the $\mathrm{K}_{19}$ cluster and $\sim 3$ electrons to the $\mathrm{K}_{24}$ strip (thus, the islands are still positive overall), and the restored electron density is concentrated near the edges (just as in classical electrostatics). The preferential accumulation of the restored electrons near the edges is the cause of the elevated beach features seen in the line scans of Figs. 4(f) and 4(h). Note that in the presence of the field, the total electronic charge on the $\mathrm{K}$ islands is distributed approximately evenly across them, but the wave function of the "beach" electrons decays more slowly into the vacuum because of the lower ionic coordination number at the island edges; this gives rise to an increased tunneling rate to the tip and thus the larger apparent height of the "bright beach" features.

Several key features of the experiment can now be understood. First, the bright beaches show up only when the STM bias is negative enough for sufficient charge restoration to take place; our simulations do not show these features with electric fields of $0.1 \mathrm{~V} / \AA$ or $0.25 \mathrm{~V} / \AA$. Second, a positive sample bias does not yield bright edges because a field pointing towards the tip depopulates the $\mathrm{K}$ islands even further of electrons. Third, a $\mathrm{K}$ atom pickup by the tip changes the shape of the field-and most likely makes it stronger locally-and hence displays bright beach features at smaller negative bias. Fourth, the second-layer $\mathrm{K}$ island cannot localize (restored) electrons at its edges, because it shares the same delocalized electron density with the (extended) K layer beneath. Finally, one can see in Fig. 4 that the overall height of the islands in STM increases with the electric field. This is in line with the original underestimation of the measured STM heights by theory without the field.

In conclusion, we expect that similar experimental features may be observed in other metallic 2D islands on flat semiconducting, semimetallic, or insulating substrates, e.g., $\mathrm{Rb}$ and $\mathrm{Cs}$ on graphite. The failure of the traditional approach to STM simulation in this finite-size system reopens the general question of including the electric field in STM modelling [30,31].

We thank the EPSRC and Academy of Finland for financial support. The calculations were run on Cray
XT4/XT5 and IBM Blue Gene/P supercomputers at CSC (Espoo, Finland) and IAS (Forschungszentrum Jülich, Germany).

[1] Z. Y. Li, K. M. Hock, and R. E. Palmer, Phys. Rev. Lett. 67, 1562 (1991).

[2] K. Rytkönen, J. Akola, and M. Manninen, Phys. Rev. B 75, 075401 (2007); 69, 205404 (2004).

[3] M.S. Dresselhaus and G. Dresselhaus, Adv. Phys. 51, 1 (2002).

[4] M. A. Sierra et al., Angew. Chem.. Int. Ed. Engl., Suppl. 41, 3442 (2002).

[5] G. Csányi et al., Nature Phys. 1, 42 (2005).

[6] J.-M. Tarascon and M. Armand, Nature (London) 414, 359 (2001).

[7] A. Lovell et al., Phys. Rev. Lett. 101, 126101 (2008).

[8] S. Patchkovskii et al., Proc. Natl. Acad. Sci. U.S.A. 102, 10439 (2005).

[9] A. Grüneis et al., Phys. Rev. Lett. 100, 037601 (2008).

[10] M. Caragiu and S. Finberg, J. Phys. Condens. Matter 17, R995 (2005).

[11] K. M. Hock, J. C. Barnard, R. E. Palmer, and H. Ishida, Phys. Rev. Lett. 71, 641 (1993).

[12] M. Breitholtz et al., Phys. Rev. B 70, 125108 (2004).

[13] H. Ishida and R. E. Palmer, Phys. Rev. B 46, 15484 (1992).

[14] F. Ancilotto and F. Toigo, Phys. Rev. B 47, 13713 (1993).

[15] D. Lamoen and B. N. J. Persson, J. Chem. Phys. 108, 3332 (1998).

[16] L. Lou, L. Österlund, and B. Hellsing, J. Chem. Phys. 112, 4788 (2000).

[17] D. Anselmetti, R. Wiesendanger, and H. J. Güntherodt, Phys. Rev. B 39, 11135 (1989).

[18] K. H. Wu et al., Phys. Rev. Lett. 91, 126101 (2003).

[19] M. Breitholtz et al., Phys. Rev. B 64, 073301 (2001).

[20] F. Yin and R. E. Palmer (to be published).

[21] K. M. Hock and R. E. Palmer, Surf. Sci. 284, 349 (1993).

[22] CRC Handbook of Chemistry and Physics, edited by David R. Lide (Internet Version 2007) (CRC Press/ Taylor and Francis, Boca Raton, Forida, USA, 2007), 87 th ed.

[23] S.-W. Hla, L. Bartels, G. Meyer, and K.-H. Rieder, Phys. Rev. Lett. 85, 2777 (2000).

[24] CPMD v3.12. Copyright IBM Corp 1990-2007, Copyright MPI für Festkörperforschung Stuttgart 1997-2001.

[25] J. P. Perdew et al., Phys. Rev. Lett. 100, 136406 (2008).

[26] N. Troullier and J.L. Martins, Phys. Rev. B 43, 1993 (1991).

[27] J. J. Mortensen, L. B. Hansen, and K. W. Jacobsen, Phys. Rev. B 71, 035109 (2005).

[28] P. E. Blöchl, Phys. Rev. B 50, 17953 (1994).

[29] M. Brack, Rev. Mod. Phys. 65, 677 (1993).

[30] W. A. Hofer, A. S. Foster, and A. L. Schluger, Rev. Mod. Phys. 75, 1287 (2003).

[31] M. W. Radny et al., Phys. Rev. B 74, 113311 (2006). 